\title{
Hounsfield Unit
}

National Cancer Institute

\section{Source}

National Cancer Institute. Hounsfield Unit. NCI Thesaurus. Code C94908.

Term given to the dimensions of the quantifier of $x$-ray attenuation, CT number. 